\title{
Study of Physical Adsorption for Ethanol Gasoline on Metal Surfaces
}

\author{
Li Na*, Han Lu, Guo Xin, Tao Zhiping, Long Jun \\ Research Institute of Petroleum Processing, Beijing, China \\ Email address: \\ lina.ripp@sinopec.com (Li Na) \\ ${ }^{*}$ Corresponding author \\ To cite this article: \\ Li Na, Han Lu, Guo Xin, Tao Zhiping, Long Jun. Study of Physical Adsorption for Ethanol Gasoline on Metal Surfaces. International Journal \\ of Oil, Gas and Coal Engineering. Vol. 8, No. 2, 2020, pp. 47-52. doi: 10.11648/j.ogce.20200802.13
}

Received: April 9, 2020; Accepted: May 13,2020; Published: May 29, 2020

\begin{abstract}
Using molecular simulation technology based on classical mechanic methods, the physical adsorption conformation of the representative conventional gasoline molecule, ethanol molecule and its oxidation intermediates, including acetaldehyde and acetic acid, on different metal surfaces was performed. Furthermore, the interaction energy composed of van der Waals and electrostatic between the absorbed molecules and the metal surfaces was calculated to study the influence of ethanol gasoline on the metal materials in comparison with the conventional gasoline. The results concluded that iron is the most likely to make strong physical adsorption with organic molecules than other surfaces, whether it is conventional gasoline molecule or ethanol molecule, or the oxidation intermediates. It may be related to the crystal configuration, coordination, atomic electron distribution and orbitals distribution of iron surface. The most stable among the studied surfaces is copper, followed by aluminum. Acid molecules, due to the presence of carboxyl group, are the most prone to form strong adsorption on the metal surfaces. The functional additives, such as antioxidant, stabilizer, detergent, dispersant or corrosion inhibitor, were critical for ethanol gasoline to avoid the undesirable influences. ESP distribution and the charges of the module molecules were calculated to make further analysis based on quantum theory.
\end{abstract}

Keywords: Ethanol Gasoline, Physical Adsorption, Metal Surface, Molecular Simulation

\section{Introduction}

During its storage in oil tank or use in the automobile engine, gasoline may be attacked by the oxygen in the air to be oxidized to produce the so-called gum [1-4]. Because of the polarity, it could stick on the metal surfaces or other materials in contact. For instance, when the gasoline molecule formed sticky gum in the oil tank, filter or pipeline, it will affect the oil supply seriously. Deposition in the intake or exhaust valves will lead to coke, which could result in valve closure lax. Deposition in the cylinder head and piston will result in cylinder poor heat radiation and even increase combustion knock tendency [5-8]. Some literatures [4, 5, 9] have demonstrated that the oxidation of hydrocarbon molecules follows the free radical chain reaction mechanism, which contains chain initiation, chain propagation and chain termination.

In order to make full use of the aged grain, fifteen ministries have issued a joint document to achieve nationwide coverage of ethanol gasoline by 2020 . The effect of the introduction of ethanol molecules on the performance of gasoline needs to be further studied. From the perspective of molecular structure, the presence of oxygen atoms in ethanol molecules may cause changes in oxidation stability. The previous literature [10] has indicated that ethanol gasoline is more prone to oxidation to produce acetic acid or acetaldehyde, than conventional gasoline, which could lead to changes in its molecular composition and physical \& chemical properties. The generation of acetic acid will lead to the stronger acidity of ethanol gasoline, which will have a certain degree of influence on engine fuel tank, oil and gas pipeline, storage tank, gas station refueling gun and other materials in contact. Further research is needed to determine whether corrosion will occur.

The general study mainly used the macroscopic test to find out whether it will get rusty or not. In fact, the metal corrosion is not only a simple macroscopic phenomenon but also a microscopic process that involves some details 
invisible to our naked eyes. It is difficult to obtain the thermodynamic and kinetic data for the process by experimental measurements [11]. In this work, molecular simulation technique was used to reveal the information at the molecular level.

The present work intends to study the adsorption behavior of conventional gasoline hydrocarbon and ethanol molecule and its oxidation intermediates to understand what influence the ethanol gasoline will have on the common used metal materials in comparison with conventional gasoline. For this purpose, the interaction energy and conformation of each absorbed molecules on metal surfaces was investigated based on molecular dynamics methods. In addition, the electrostatic potential (i.e. ESP) distribution and charges of all the studied molecules was calculated based on density functional theory (DFT). With the results, the physical adsorption of ethanol gasoline was revealed furthermore.

\section{Computational Details and Models}

\subsection{Computational Details}

Computational results were performed with molecular dynamics methods by Forcite Plus in the Material Studio 2017R2 software developed by Daussault Biovia Inc. Geometry optimizations and the ESP distribution of all molecules were carried out with GGA-PBE functional employing basis set DNP by Dmol ${ }^{3}$ module [12-14] based on DFT method. SCF calculations were converged tightly (SCF tolerance: $1 \times 10^{-6} \mathrm{Ha}$; energy: $1 \times 10^{-5} \mathrm{Ha} ; \max$ force: $0.0002 \mathrm{Ha} / \mathrm{nm}$; max displacement: $5 \times 10^{-4} \mathrm{~nm}$ ).

The initial metal lattices were derived from the structural database of MS software. The simulation of the interaction between the molecules and the surfaces is carried out in a simulation box $(9.9 \AA \times 9.9 \AA \times 21.9 \AA)$ with periodic boundary conditions to model a representative part of the interface devoid of any arbitrary boundary effects. During molecular simulations, the metal systems were all kept "frozen", and the absorbed molecules were allowed to interact with the metal surfaces freely.

It was found that $\mathrm{Cu}(111), \mathrm{Fe}(110)$ and $\mathrm{Al}$ (111) were the most stable surfaces of the corresponding crystal structure. The selected surfaces in the paper were shown in figure 1 .

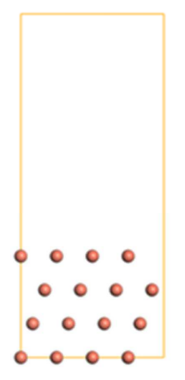

(a) $\mathrm{Cu}(111)$

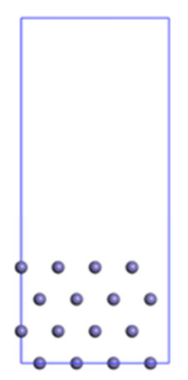

(b) $\mathrm{Fe}(110)$

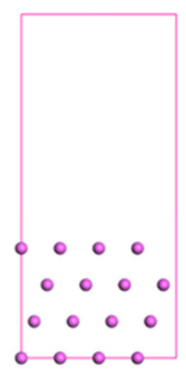

(c) $\mathrm{Al}(111)$
Figure 1. Structure of selected metal surfaces.

The interaction energy, $E_{\text {interaction }}$ between the metal surface and the adsorbed molecule is calculated according to the following equation (1) [15]:

$$
E_{\text {interaction }}=E_{\text {total }}-\left(E_{\text {surface }}+E_{\text {molecule }}\right)
$$

In which $E_{\text {total }}$ is the total energy of the simulation system, $E_{\text {surface }}$ is the energy of metal surface, and $E_{\text {molecule }}$ is the energy of the stabilized absorbed molecules.

\subsection{Model Compounds}

As is well known that gasoline is a mixture mainly composed of olefins, paraffins, naphthenes and aromatic hydrocarbons whose carbon number was $4 \sim 12$. But in this work, in order to make the metal surfaces consistent, ethane whose carbon number is as same as ethanol was selected as the representative conventional gasoline model compound, to study the differences in adsorption properties, between ethanol gasoline and conventional gasoline molecules. Studies have shown that ethanol gasoline is prone to make oxidation reaction, and during which the ethanol molecule was oxidized through different reaction paths to produce some key intermediates such as acetaldehyde or acetic acid. The structure of model compounds was shown in figure 2 .

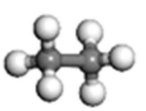

(a) Conventional gasoline

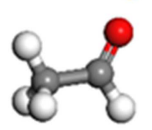

(c) Acetaldehyde

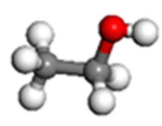

(b) Ethanol

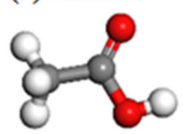

(d) Acetic acid
Figure 2. Structure of model compounds in ethanol gasoline.

\section{Results and Discussion}

Adsorption is the adhesion of a gas or solute on a solid or liquid surface. The chemisorption process has the formation and destruction of chemical bonds, whose adsorption heat is relatively large, and need to be carried out under high temperature. This process is selective. Physical adsorption is the adsorption caused by intermolecular interaction without selectivity which is generally carried out at low temperature, low speed. And the adsorption heat is relative lower. Here the physical adsorption behavior will be studied based on molecular dynamics method and then further analysis will be made with quantum method i.e. DFT theory.

\subsection{Calculations}

\subsubsection{Conventional Gasoline}

After molecular dynamics simulation, the physical adsorption equilibrium conformation of conventional gasoline molecule and ethanol and its oxidation intermediates on different metal surfaces was carried out shown in figure 3 to figure 6. As can be seen from figure 3, the ethane molecules were all adsorbed on the three metal surfaces in a 
horizontal and tiled form without chemical interaction. Moreover, the distance from the absorbed molecule to the three metal surfaces is relatively close.

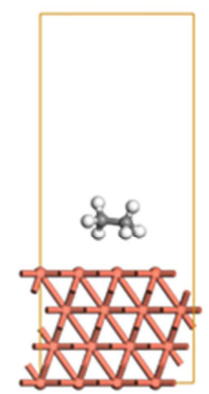

(a) $\mathrm{Cu}$

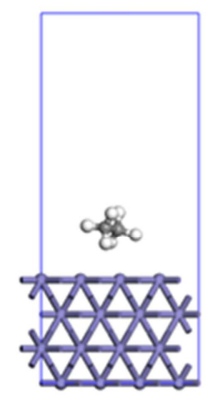

(b) $\mathrm{Fe}$

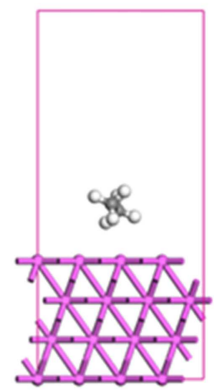

(c) Al
Figure 3. Physical adsorption conformation of conventional gasoline on metal surfaces.

Furthermore, the adsorption energy was calculated. The results were listed in table 1 . It was indicated that the interaction energy of ethane and iron surface was the biggest, followed by copper and aluminum surfaces. From a numerical point of view, the adsorption energy on iron surface was $-99.93 \mathrm{~kJ} / \mathrm{mol}$, which is mainly contributed by van der Waals force $(-96.97 \mathrm{~kJ} / \mathrm{mol})$. The corresponding electrostatic energy is relatively few $(-2.95 \mathrm{~kJ} / \mathrm{mol})$, although it is the strongest value among the three metal surfaces. The other two total interaction energy were both composed of van der Waals and electrostatic, which were $-50.86 \mathrm{~kJ} / \mathrm{mol}$ and $-44.94 \mathrm{~kJ} / \mathrm{mol}$ respectively.

Table 1. Interaction energy of physical adsorption for conventional gasoline (Unit: kJ/mol).

\begin{tabular}{llll}
\hline Interaction energy & $\mathbf{C u}$ & $\mathbf{F e}$ & $\mathbf{A l}$ \\
\hline$E_{\text {Van der Waals }}$ & -49.17 & -96.97 & -43.29 \\
$E_{\text {electrostatic }}$ & -1.69 & -2.95 & -1.66 \\
$E_{\text {total }}$ & -50.86 & -99.93 & -44.94 \\
\hline
\end{tabular}

Conventional gasoline molecules will also have a certain adsorption effect on different metal surfaces. Even if it is physical adsorption, the adsorption capacity of ethane molecules on iron surface cannot be ignored. With the continuous wash and immersion of gasoline, the metal surface will inevitably be corrosion to a certain extent.

\subsubsection{Ethanol}

After MD calculation, the adsorption conformation of ethanol molecule on each metal surface was obtained shown in figure 4. It can be seen from that the distance between the gasoline molecule and the metal surface is also far away without chemical bond formed. The skeleton of the ethanol molecule and its hydroxyl group at the tail both laid on the metal surfaces with as much contacted area as possible. The adsorption conformation of the ethanol molecule is very similar.

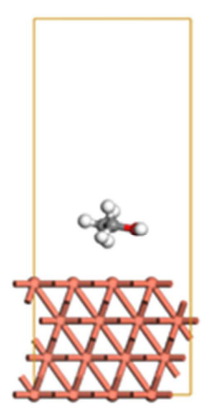

(a) $\mathrm{Cu}$

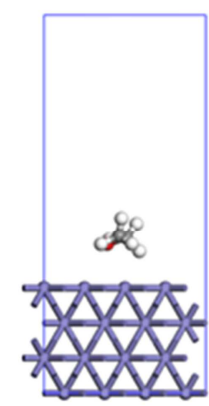

(b) $\mathrm{Fe}$

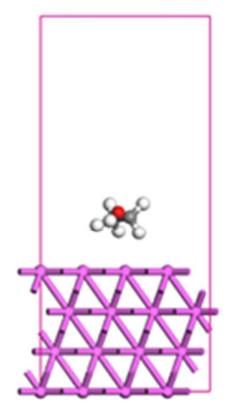

(c) $\mathrm{Al}$
Figure 4. Physical adsorption conformation of ethanol molecule on metal surfaces.

The interaction energy of ethanol molecule on each surface was calculated. The results were listed in table 2 . In contrast, among the three metal surfaces, the adsorption capacity of ethanol molecule on iron surface was also the strongest which was the same as that of ethane molecule. It may be related with the specific crystal structure of the iron surface which was more likely to make adsorption effect with the organic molecules.

Specifically, it was indicated from the values in table 2 , the adsorption energy between ethanol molecule and metal surfaces was also contributed by van der Waals. In addition, the interaction energy of physical adsorption for ethanol on different metal surfaces was about $10 \sim 30 \mathrm{~kJ} / \mathrm{mol}$ higher than ethane molecule, which may depend on the hydroxyl group in ethanol molecule. The higher part than ethane molecule is also mainly provided by van der Waals.

Thus, due to the introduction of ethanol to gasoline, the effect of ethanol gasoline on metal surfaces was more seriously than the conventional gasoline, to which some attention should be given during the storage, transportation and use in engine.

Table 2. Interaction energy of physical adsorption for ethanol (Unit: $\mathrm{kJ} / \mathrm{mol}$ ).

\begin{tabular}{llll}
\hline Interaction energy & $\mathbf{C u}$ & $\mathbf{F e}$ & $\mathbf{A l}$ \\
\hline$E_{\text {van der Waals }}$ & -56.62 & -118.42 & -53.08 \\
$E_{\text {electrostatic }}$ & -2.08 & -3.68 & -2.10 \\
$E_{\text {total }}$ & -58.71 & -122.10 & -55.17 \\
\hline
\end{tabular}

\subsubsection{Acetaldehyde}

It has been reported that acetaldehyde is one of the important oxidation intermediates of ethanol. This substance is inevitably produced during the use of ethanol gasoline, so when ethanol gasoline comes into contact with the metal surface, there must be trace amounts of acetaldehyde molecules interacting with the metal surface at the same time.

With the same calculation method, the physical adsorption conformation of acetaldehyde on metal surfaces was given in figure 5. Similar to ethanol molecule, the oxygen-containing functional group in acetaldehyde molecule, i.e. the aldehyde group, is as close to the metal surface as possible, leading to the final stable adsorption conformation. 


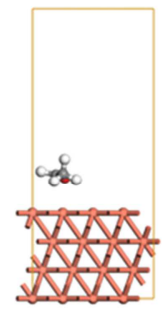

(a) $\mathrm{Cu}$

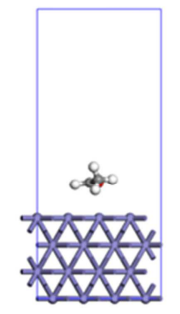

(b) $\mathrm{Fe}$

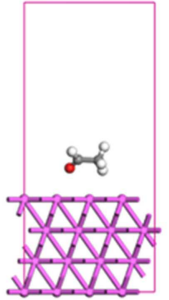

(c) $\mathrm{Al}$
Figure 5. Physical adsorption conformation of acetaldehyde on metal surfaces.

The specific adsorption values were calculated in table 3 . It could be obviously found that the interaction energy of physical adsorption for acetaldehyde on the studied metal surfaces more approach to that of ethanol. However, the interaction of acetaldehyde on copper or iron surface was just few lower than ethanol, which may have something to do with the fact that the acetaldehyde molecule has two fewer hydrogen atoms than the ethanol molecule.

Table 3. Interaction energy of physical adsorption for acetaldehyde (Unit: $\mathrm{kJ} / \mathrm{mol}$ ).

\begin{tabular}{llll}
\hline Interaction energy & $\mathbf{C u}$ & $\mathbf{F e}$ & $\mathbf{A l}$ \\
\hline$E_{\text {van der Waals }}$ & -46.05 & -117.44 & -57.43 \\
$E_{\text {electrostatic }}$ & -2.04 & -3.59 & -2.05 \\
$E_{\text {total }}$ & -48.09 & -121.04 & -59.49 \\
\hline
\end{tabular}

For physical adsorption, the size of the interaction area or the number of interaction sites may be critical to the adsorption strength. And on the other hand, unlike ethane molecule, both ethanol and acetaldehyde molecules both contain oxygen atoms, which are known to be more electronegative than carbon or hydrogen atoms, making the molecules more polar. This may be another important factor for strong adsorption.

\subsubsection{Acetic Acid}

At the same time, it has been pointed out that acetic acid is a key oxidation product in the further oxidation of ethanol molecule, and its molecule contain acidic carboxyl groups, so it is necessary to study the physical adsorption behavior of acetic acid molecules on different metal surfaces. It will provide theoretical basis for the safe and reliable circulation and use of ethanol gasoline.

With MD method, the physical adsorption conformation of acetic acid on metal surfaces was listed in figure 6 .

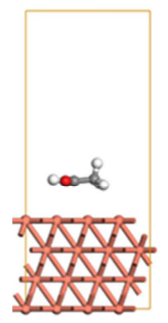

(a) $\mathrm{Cu}$

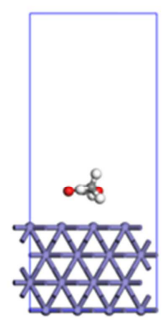

(b) $\mathrm{Fe}$

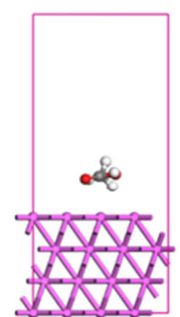

(c) $\mathrm{Al}$
Figure 6. Physical adsorption conformation of acetic acid on metal surfaces.
It was demonstrated that the skeleton of the acetic acid molecule, especially the carboxyl functional group, is almost tiled adsorbed on the metal surfaces. In order to maintain the conformation in space, the hydrogen atoms in the molecule almost remain in their own structural shape, which is not in full contact with the metal surfaces. At present, the physical adsorption is studied, and the interaction between the electrons at the chemical level is not considered. The physical adsorption conformation of acetic acid molecules on different metal surfaces is particularly similar.

The physical adsorption energy of acetic acid molecules on different metal surfaces was listed in table 4. By comparison, the interaction energy of acetic acid on the iron surface is the highest, among which, the van der Waals interaction energy is $-150.84 \mathrm{~kJ} / \mathrm{mol}$ and the electrostatic energy is $-4.26 \mathrm{~kJ} / \mathrm{mol}$. However, the adsorption energy of acetic acid on the copper or aluminum surfaces is $-80.89 \mathrm{~kJ} / \mathrm{mol}$ and $-70.47 \mathrm{~kJ} / \mathrm{mol}$ respectively, among which the electrostatic energy also account for a small share.

Table 4. Adsorption energy of acetic acid on metals (Unit: $\mathrm{kJ} / \mathrm{mol}$ )

\begin{tabular}{llll}
\hline Interaction energy & $\mathbf{C u}$ & $\mathbf{F e}$ & $\mathbf{A l}$ \\
\hline$E_{\text {van der Waals }}$ & -77.89 & -150.84 & -68.02 \\
$E_{\text {electrostatic }}$ & -2.41 & -4.26 & -2.45 \\
$E_{\text {total }}$ & -80.31 & -155.10 & -70.47 \\
\hline
\end{tabular}

As to the physical adsorption of the same molecule on different metals, the adsorption interaction on the iron surface is stronger than that on other surfaces, whether it is the conventional gasoline molecule, the ethanol molecule or its oxidation intermediates. Thus, iron surface is the most vulnerable to oil corrosion. Iron materials should be avoided for oil system. In addition, with the deepening oxidation of ethanol gasoline, the effect of oxidation intermediates on metal surface is stronger than conventional gasoline molecules or even ethanol molecule. Therefore, the oxidation stability of ethanol gasoline should be paid more attention to.

\subsection{Quantum Calculations}

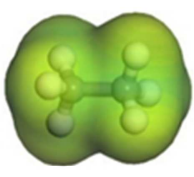

(a) Conventional Gasoline

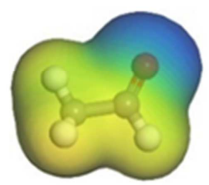

(c) Acetaldehyde

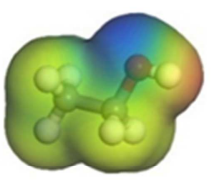

(b) Ethanol

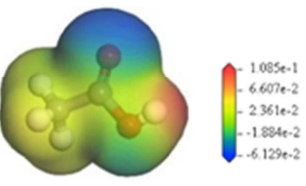

(d) Acetic acid
Figure 7. The ESP distribution of the absorbed molecules.

The physical adsorption between oil molecules and metal surfaces produces a weak interaction. The adsorption conformation and capacity depend on the structure and 
properties of the adsorbed molecules when the metal surfaces are the same. The ESP (electrostatics potential) distribution on the absorbed molecules is one of the key factors affecting the weak interaction. Thus, the ESP distribution of the adsorbed molecules was obtained by the property analysis with $\mathrm{Dmol}^{3}$ program, shown in figure 7.

The electrostatic potential values of different surface areas are shown by different colors. As indicated by the color ruler, blue represents the negative charge region. It can be seen from that the electronegativity of ethanol or its oxidation intermediates is mainly distributed on the $\mathrm{O}$ atoms at the terminal functional groups such as the hydroxyl, the aldehyde or the carboxyl group. While the electronegativity of the conventional gasoline molecule is not obvious.

The ESP charge distribution of the absorbed molecules was calculated in figure 8. It was indicated that the ESP charge of $\mathrm{C}$ atoms in conventional gasoline molecule is $-0.117 \mathrm{e}$ and the charge of $\mathrm{H}$ atoms is around $0.038 \mathrm{e}$. However, the ESP charge of $\mathrm{O}$ atoms in ethanol or its oxidation intermediates is $-0.614 \mathrm{e},-0.447 \mathrm{e},-0.518 \mathrm{e}$ and $-0.545 \mathrm{e}$ respectively. And the charge of the corresponding connected $\mathrm{C}$ atoms is at around $0.5 \sim 0.7 \mathrm{e}$.

It was known that the lower the ESP, the higher the active molecule is $[16,17]$. The strength of $\mathrm{O}$ atom sites in ethanol or the oxidation intermediates is stronger than the atoms in conventional gasoline molecule. It could demonstrate that the adsorption interaction energy of the ethanol gasoline on metal surfaces would be significantly higher than that of conventional gasoline.

Although the strength of oxygen atom in ethanol was stronger than that in acetaldehyde or acetic acid, there are two active sites in acetic acid. The effect of acetic acid on the metal surface is significantly stronger. However, the carbonyl group in acetaldehyde is easier to tile on the metal surfaces than the hydroxyl group in ethanol, so the ultimate performance is not much different.

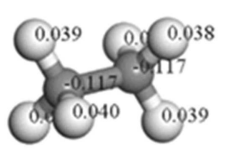

(a) Conventional Gasoline

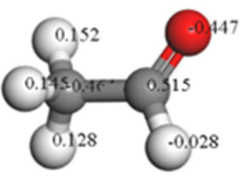

(c) Acetaldehyde

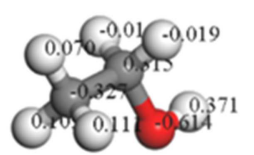

(b) Ethanol

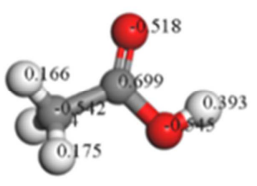

(d) Acetic acid
Figure 8. The ESP charge distribution of the absorbed molecules.

According to previous studies, ethanol gasoline is more prone to oxidation than conventional gasoline to produce molecules with higher polarity such as aldehydes or acids. Studies have shown that the ESP charge distribution of the molecule is more uneven and more likely to adsorb on the metal surfaces. Then corrosion and other adverse effects on metal materials may damage the nature of the material itself or even make it unable to play the normal performance. It is a potential risk for the normal use of the fuel gun, storage tank, fuel tank, engine intake systems which the ethanol gasoline usually contact with. On the other hand, due to the oxidation of ethanol, the oxidation chain radical reaction of conventional gasoline molecules may be triggered, and the oxidation intermediates of ketones, aldehydes or acid with larger molecular weight will also be produced, thus worsening the performance of gasoline itself.

\section{Conclusion}

Using molecular simulation methods, the physical adsorption behavior of conventional gasoline and ethanol gasoline on different metal surfaces was investigated which also considered the oxidation intermediates of ethanol molecule. According to the interaction conformation and energy results, it was indicated that the skeleton of the adsorbed molecule and its functional groups are basically spread out adsorbed on the metal surfaces.

In the studied metals, iron is the most likely to make strong physical adsorption with molecules than other surfaces, whether it is conventional gasoline molecule or ethanol gasoline molecules. It may be related to the crystal configuration, coordination, atomic electron distribution and orbitals distribution of iron surface. The most stable among the studied surfaces is copper, followed by aluminum. With the vehicle lightweight development in the future, aluminum is a reference choice in terms of the weight and stability.

In the oxidation intermediates, due to the presence of carboxyl group, acid molecule was the most prone to form relative strong adsorption on the metal surfaces. It was necessary for ethanol gasoline to add corrosion inhibitors to avoid the undesirable phenomenon. The physical adsorption energy of aldehyde or ethanol molecules was few higher than that of conventional gasoline, but almost at the same level. Therefore, for ethanol gasoline, we should pay more attention to its oxidation stability, to avoid further oxidation reactions of ethanol molecule. Thus, the functional additives, especially antioxidant, stabilizer, detergent dispersant or corrosion inhibitor, were critical for ethanol gasoline to make regular service in storage tank or engine systems.

\section{Acknowledgements}

This work was supported financially by SINOPEC research project (No. 119026-2), the National Key Research and Development Program of China (No. 2017YFB0306505) and SINOPEC research project (No. 118023-1).

\section{References}

[1] Nagpal JM, Joshi GC, Singh J, Kumar K. Studies on the nature of gum formed in cracked naphtas [J]. Oxid Commun 1998, 21 (4): 468-477. 
[2] Xue Dong, Yachao Chang, Bo Niu, et al. Development of a practical reaction model of polycyclic aromatic hydrocarbon (PAH) formation and oxidation for diesel surrogate fuel [J]. Fuel, 2020, 267: 117159.

[3] Kinoshita M, Saito A, Matsushita S, Shibata H, Niwa Y. Study of deposit formation mechanism on gasoline injection nozzle [C]. SAE paper 1998, 19: 355-357.

[4] Li Na, Long Jun, Zhao Yi, et al. Molecular Simulation of the Mechanism of Oxidation Gum Formation of Typical Gasoline Hydrocarbon [J]. Acta Petrolei Sinica (Petroleum Processing Section), 2018, 34 (2): 354-364.

[5] Li Na, Long Jun, Zhao Yi, Tao Zhiping, Dai Zhenyu. DFT study of oxidation initiation for different compound in gasoline [J]. Journal of Clean Energy Technologies, 2017, 6 (3): 242-245

[6] Paul Lacey, Sandro Gail, Jean Marc Kientz, et. al. Internal Fuel Injector Deposits [C]. SAE Paper 2011-01-1925.

[7] Akio Tanaka, Koichi Yamada, Toshihiko Omori, et. al. Inner Diesel Injector Deposit Formation Mechanism [C]. SAE Paper, 2013-01-2661.

[8] Ji Xiang, Song Yingjin, Liu Rui, et al. Effect of oxidation decay of alcohol fuel engine on exhaust emission [J]. Journal of Harbin University of Commerce (Natural Sciences Edition), 2018, 34 (5): 573-576.

[9] Nicholas J. Kuprowicz, Steven Zabarnick, Zachary J. West, et. al. Use of Measured Species Class Concentrations with Chemical Kinetic Modeling for the Prediction of Autoxidation and Deposition of Jet Fuels [J]. Energy \&Fuels 2007, 21, 530-544.

[10] Li Na, Han Lu, Guo Xin, et al. DFT study of oxidation reaction paths for ethanol gasoline [J]. Journal of Energy and Natural Resources, accepted.

[11] Ruben Epping, Stefanie Kerkering, Jan T. Andersson. Influence of Different Compound Classes on the Formation of Sediments in Fossil Fuels During Aging [J]. energy\& fuels, 2014, 28, 5649-5656.

[12] Delley B. From molecules to solids with the DMol3 approach [J]. J Chem Phys, 2000, 113 (18): 7756-7764.

[13] Kohn W, Sham L J. Self-consistent equations including exchange and correlation effects [J]. Phys Rev, 1965, 140 (4A): A1133-A1138.

[14] J. P. Perdew, K. Burke, M. Ernzerhof, Generalized gradient approximation made simple, Phys. Rev. Lett. 1996, 77: 3865-3868.

[15] Saha S K, Dutta A, Ghosh P, et al. Novel Schiff-base molecules as efficient corrosion inhibitors for mild steel surface in $1 \mathrm{M} \mathrm{HCl}$ medium: experimental and theoretical approach [J]. Phys Chem Chem Phys, 2016, 18: 17898-179.

[16] Li X, Deng S, Fu H, et al. Adsorption and inhibition effect of 6-benzylaminopurine on cold rolled steel in $1.0 \mathrm{M} \mathrm{HCl}[\mathrm{J}]$. Electrochim Acta, 2009, 54 (16): 4089-4098.

[17] Mishra P M. Electron affinity calculation for selected PAHs using DFT: Effect of cyclopenta ring fusion and aromaticity [J]. Comput Theor Chem, 2015, 1068: 165-171. 